\title{
Stabilisation of time delay systems with nonlinear disturbances using sliding mode control
}

\author{
Adrian E. Onyeka and Xing-Gang Yan* \\ School of Engineering and Digital Arts, \\ University of Kent, \\ Canterbury, CT2 7NT, UK \\ Email: aeo20@kent.ac.uk \\ Email: x.yan@kent.ac.uk \\ *Corresponding author

\section{Zehui Mao} \\ College of Automation Engineering, \\ Nanjing University of Aeronautics and Astronautics, \\ Nanjing, 210016, China \\ Email: zehuimao@nuaa.edu.cn
}

\section{Jianqiu Mu}

B-535 Zhongchuang Space,

Huilongguan East Street,

Changping, Beijing, 102200, China

Email:jm838@kent.ac.uk

\begin{abstract}
This paper focuses on a class of control systems with delayed states and nonlinear disturbances using sliding mode techniques. Both matched and mismatched uncertainties are considered which are assumed to be bounded by known nonlinear functions. The bounds are used in the control design and analysis to reduce conservatism. A sliding function is designed and a set of sufficient conditions is derived to guarantee the asymptotic stability of the corresponding sliding motion by using the Lyapunov-Razumikhin approach which allows large time varying delay with fast changing rate. A delay dependent sliding mode control is synthesised to drive the system to the sliding surface in finite time and maintain a sliding motion thereafter. Effectiveness of the proposed method is demonstrated via a case study on a continuous stirred tank reactor system.
\end{abstract}

Keywords: stabilisation; sliding mode control; time delay; Lyapunov-Razumikhin approach; uncertain systems.

Reference to this paper should be made as follows: Onyeka, A.E., Yan, X-G., Mao, Z. and $\mathrm{Mu}$, J. ( $\mathrm{xxx})$ 'Stabilisation of time delay systems with nonlinear disturbances using sliding mode control', Int. J. Modelling, Identification and Control, Vol. X, No. Y, pp.xxx-xxx.

Biographical notes: Adrian E. Onyeka received his HND and BTech in the Federal Polytechnic Nekede and Federal University of Technology, respectively in Owerri, Nigeria. He obtained his MSc in Electronics and Electrical Engineering in the Anglia Ruskin University, Cambridge UK in 2014. Prior to that, he has worked as an Academic at the Imo state University, Owerri Nigeria. Currently, he is a PhD candidate in the School of Engineering and Digital Arts, University of Kent, Canterbury, UK. His research interest includes variable structure control for time delay systems and decentralised power system control.

Xing-Gang Yan received his BSc degree from Shaanxi Normal University, in 1985, his MSc degree from Qufu Normal University in 1991, and his PhD from Northeastern University, China in 1997. Currently, he is a Senior Lecturer at the University of Kent, UK. He was a Lecturer in Qingdao University, China from 1991 to 1994. He worked as a Research Fellow or Research Associate in China, Singapore and UK. His research interests include sliding mode control and decentralised control. He is a co-author of over 160 referred papers including three books.

Zehui Mao received her PhD in Control Theory and Control Engineering from the Nanjing University of Aeronautics and Astronautics, Nanjing, China, in 2009. She is now an Associate Professor at the College of Automation Engineering in the Nanjing University of Aeronautics and Astronautics, China. And she was a Visiting Scholar in the University of Virginia. She 
worked in the areas of fault diagnosis, with particular interests in nonlinear control systems, sampled-data systems and networked control systems. Her current research interests include fault diagnosis and fault-tolerant control of systems with disturbance and incipient faults, and high speed train and spacecraft flight control applications.

Jianqiu $\mathrm{Mu}$ received BEng in Electrical Engineering and Automation from the Chongqing University, Chongqing, China, in 2012, MSc (distinction) in Advanced Electronic System Engineering and $\mathrm{PhD}$ in Electronic Engineering from the University of Kent, Canterbury, UK, in 2013 and 2018 respectively. His current research interests include sliding mode control, decentralised control, nonlinear control systems, mobile robots and autonous vehicles.

This paper is a revised and expanded version of a paper entitled 'Sliding mode control of time-delay systems with delayed nonlinear uncertainties', presented at 20th World Congress of the International Federation of Automatic Control (IFAC), Toulouse, France, 9-14 July 2017.

\section{Introduction}

Over the past decades, time delay systems as often referred to as after-effect systems have been an active area of research in a wide range of natural and social sciences. They belong to a class of functional differential equations, existing widely in the practical world, and are mostly encountered in numerous engineering systems such as electrical networks, chemical reactors, and hydraulic, pneumatic and manufacturing processes to mention but a few (Gu et al., 2003; Richard, 2005; Zhao et al., 2015). Time delay is usually a source of instability and performance degradation in control systems which needs to be considered seriously in design, and as such, has received considerable attention over the past years, see, e.g., Richard (2003), Yan et al. (2014a) and references therein.

Motivated by recent development in large-scale system design, multiple or large time delay arise in systems due to vast network complexity. As a result, various techniques have been developed in trying to circumvent the effect of uncertain dynamical time delay systems (Richard, 2003; Yan et al., 2014a; Chen et al., 2017). Linear matrix inequality (LMI) techniques are applied in Ramakrishnan and Ray (2015) and Wang et al. (2016), but require that the considered systems are linear and the nonlinear uncertainty satisfy linear growth condition. Lyapunov-Razumikhin technique is applied in this paper which focuses on developing a robust system with large time delay and fast change rate, which has improved the requirement of the bounds on the delays compared with recent results (Gao et al., 2014; Qi et al., 2017; Jia et al., 2017). In addition, the design parameters can be obtained via LMI techniques systematically. It should be noted that one method which has proved very effective in dealing with uncertainties in the system is the sliding mode control due to its strong robustness properties against parametric uncertainties and external disturbances in the input channel, as well as its attractive features such as fast and good transient response (Zhen et al., 2014; Edwards and Spurgeon, 1998; Mu et al., 2017).

Due to its high robustness, sliding mode control has been extended to time delay systems with disturbances, and most of the existing results are in combination with other techniques such as LMI (Wu et al., 2014), optimal control
(Nikkhah et al., 2006), adaptive control (Baek et al., 2016), decentralised control (Yan et al., 2014b), where the common goal is to present less conservative conditions to guarantee high performance of systems considered. It should be noted that sliding mode control techniques can also be used to deal with mismatched uncertainties (Ghabi, 2018; Yan et al., 2017).

The problem of sliding mode control for uncertain time delay systems has been a continuous area of interest and development. Recent work carried out in this area involved different technique when compared with traditional sliding mode control, e.g., integral sliding surface where the reaching phase is eliminated (Cao and $\mathrm{Xu}, 2004$; Vaidyanathan and Rhif, 2017), are different from the usual or conventional sliding surface which has a reaching phase in Edwards and Spurgeon (1998) or that it only considered matched uncertainty (Nikkhah et al., 2006), while in Hua et al. (2008) and Xu et al. (1997) it is required that the bounds on the uncertainties satisfy the linear growth condition. Moreover, two main techniques based on Lyapunov-Krasovskii functional and Lyapunov-Razumikhin function have been largely used to deal with time delay.

It should be noted that sliding mode control for time delay with nonlinear disturbances has been studied in Yan et al. (2010) where static output feedback was considered, which has strong limitation on the system including the bounds on the uncertainties. Cao and $\mathrm{Xu}$ (2004) proposed the robust sliding mode control of nonlinear uncertain systems by analysing the lump estimated disturbances via a disturbance observer. Although static output feedback or observer-based output feedback controllers have certain advantages in real implementation, strong limitations is unavoidably required. In reality, sometimes, all the system states for example, the position and speed of a mechanical system, may be available and thus state feedback will be possible, which will largely reduce the limitation of the considered system. Moreover, the bounds on uncertainty can be much relaxed when compared with output feedback design scheme. Optimal guaranteed cost sliding mode control of interval type-2 fuzzy time-delay systems was proposed in $\mathrm{Li}$ et al. (2017) where Lyapunov-Krasovskii technique was used to analyse the delay bound. However, due to evolving complex systems, developing large time 
delay bound may be necessary. It should be noted that the Lypunov-Krasovskii method usually requires that the time varying delay is differentiable and there is limitations on the rate of change of time delay. Hence, Lyapunov-Razumikhin method which is used for large constant and time varying delay is used to circumvent this effect.

This work proposes a sliding mode control scheme for a class of time delay control systems with nonlinear delayed disturbances. The assumptions for nonlinear terms are imposed on the transformed systems to avoid unnecessary conservatism caused by coordinate transformation in theoretical analysis. Lyapunov-Razumikhin approach is used to derive a set of conditions to guarantee that the derived sliding motion is asymptotically stable in the presence of time delay. Then under assumption that all the system states are accessible, sliding mode control is synthesised such that the controlled system is driven to the sliding surface in finite time and maintains sliding motion thereafter. Case study on a continuous stirred tank reactor (CSTR) is provided to show the feasibility of the developed results and the effectiveness of the proposed method. The main contribution is summarised as follows:

1 The known bounds on the uncertainties are fully applied in the controller design to reject the effects of the uncertainties on system performance.

2 Compared with associated existing work, the proposed approach not only allows the bounds on the uncertainties have more general nonlinear form but all the design parameters relating to the sliding motion can be obtained using LMI techniques.

3 Both matched and mismatched uncertainties are considered and the bounds on matched and mismatched uncertainties involve time varying delay.

4 There is no limitation to the change rate of the time-varying delay which allows fast changing rate of the time varying delay.

\section{Preliminaries}

First, recall some basic linear system theory. Consider a linear system

$$
\dot{x}=A x+B u
$$

where $x \in \mathfrak{R}^{n}, u \in \mathfrak{R}^{m}$ are states and inputs respectively, with $m<n$. The matrix pair $(A, B)$ is of appropriate dimensions whereas $B$ is of full rank. Assume that the matrix pair $(A, B)$ is controllable. Then from basic matrix theory, it can be shown that a coordinate transformation $z=T x$ exists such that the matrix pair $(A, B)$ in the new coordinates $z$ has the following structure (Edwards and Spurgeon, 1998; Yan et al., 2017):

$$
\tilde{A}=\left[\begin{array}{ll}
A_{11} & A_{12} \\
A_{21} & A_{22}
\end{array}\right], \tilde{B}=\left[\begin{array}{c}
0 \\
B_{2}
\end{array}\right]
$$

where $A_{11} \in \mathfrak{R}^{(n-m) \times(n-m)}$, and $B_{2} \in \mathfrak{R}^{m \times m}$ is non-singular. It should be noted that such a transformation can be obtained systematically using matrix theory. Further, from Edwards and Spurgeon (1998), the fact that $(A, B)$ is controllable implies that $\left(A_{11}, A_{12}\right)$ is controllable, and thus there exists a matrix $M \in \mathfrak{R}^{m \times(n-m)}$ such that the matrix $A_{11}-A_{12} M$ is Hurwitz stable. In order to deal with time delay systems, the following well-known Razumikhin theorem is required.

Consider a time-delay system

$$
\dot{x}(t)=f(t, x(t-d(t)))
$$

with an initial condition

$$
x(t)=\phi(t), \quad t \in[-\bar{d}, 0]
$$

where $f: \mathfrak{R}^{+} \times C_{[-\bar{d}, 0]} \mapsto \mathfrak{R}^{n}$ takes $\mathfrak{R} \times($ bounded sets of $\left.C_{[-\bar{d}, 0]}\right)$ into bounded sets in $\mathfrak{R}^{n} ; d(t)$ is the time-varying delay and $\bar{d}:=\sup _{t \in \mathfrak{R}^{+}}\{d(t)\}<\infty$.

Lemma 1: [Razumikhin Theorem, Gu et al. (2003)]

If there exist class $\mathcal{K}_{\infty}$ functions $\gamma_{i}(\cdot)$ with $i=1,2$, a class $\mathcal{K}$ function $\gamma_{3}(\cdot)$ and a continuous function $V_{1}(\cdot):[-\bar{d}, \infty] \times \mathfrak{R}^{n} \mapsto \mathfrak{R}^{+}$satisfying

$$
\gamma_{1}(\|x\|) \leq V_{1}(t, x) \leq \gamma_{2}(\|x\|), t \in[-\bar{d}, \infty], x \in \mathfrak{R}^{n}
$$

such that the time derivative of $V_{1}$ along the solution of system (3) satisfies

$$
\dot{V}_{1}(t, x) \leq-\gamma_{3}(\|x\|)
$$

whenever

$$
V_{1}(t+\theta, x(t+\theta)) \leq V_{1}(t, x(t))
$$

for any $\theta \in[-\bar{d}, 0]$, then the system (3) is uniformly stable. If, in addition, $\gamma_{3}(\tau)>0$ for $\tau>0$, and there exists a continuous non-decreasing function $\gamma_{4}(\cdot)$ which satisfies $\gamma_{4}(\tau)>\tau$ for $\tau>0$ such that inequality (6) is strengthened to

$$
\dot{V}_{1}(t, x) \leq-\gamma_{3}(\|x\|)
$$

whenever

$$
V_{1}(t+\theta, x(t+\theta)) \leq \gamma_{4}\left(V_{1}(t, x(t))\right)
$$

for any $\theta \in[-\bar{d}, 0]$, then system (3) is uniformly asymptotically stable.

Lemma 2: (see Yan et al., 2012)

Let the matrix $N_{1} \in \mathfrak{R}^{m \times n}$ and vectors $x \in \mathfrak{R}^{m}$ and $y \in \mathfrak{R}^{n}$. Then, the inequality

$$
x^{T} N_{1} y \leq \frac{1}{2 \epsilon} x^{T} N_{1} N_{2}^{-1} N_{1}^{T} x+\frac{\epsilon}{2} y^{T} N_{2} y
$$


holds for any symmetric positive-definite matrix $N_{2} \in \mathfrak{R}^{n \times n}$ and any positive constant $\epsilon$.

The results above will be used in the subsequent analysis.

\section{Problem formulation}

Consider a time varying delay system with delayed disturbance described by

$$
\dot{x}=A x+A_{d} x_{d}+B\left(u+\bar{G}\left(t, x, x_{d}\right)\right)+\bar{F}\left(t, x, x_{d}\right)
$$

where $x \in \Omega \subset \mathfrak{R}^{n}(\Omega$ is an neighbourhood of the origin), $u \in \mathfrak{R}^{m}$ are the states and inputs respectively; $A, A_{d} \in \mathfrak{R}^{n \times n}$ and $B \in \mathfrak{R}^{n \times m}(m<n)$ are constant matrices with $B$ being of full rank. The vectors $\bar{G}(\cdot)$ and $\bar{F}(\cdot)$ represent the matched and mismatched disturbances affecting the system respectively. The symbol $x_{d}:=x(t-d)$ represents the delayed state where $d:=d(t)$ is the time varying delay which is assumed to be known, continuous, non-negative and bounded in $\mathfrak{R}^{+}:=\{t \mid t \geq 0\}$, that is

$$
\bar{d}:=\sup _{t \in \mathfrak{R}^{+}}\{d(t)\}<\infty
$$

The initial condition related to the time delay is given by

$$
x(t)=\Phi(t), \quad t \in[-\bar{d}, 0]
$$

where $\Phi(\cdot)$ is continuous in $[-\bar{d}, 0]$. It is assumed that all the nonlinear functions are smooth enough for the subsequent analysis, which guarantees that the unforced system has unique continuous solutions.

In this paper, the objective is to design a sliding mode control for the system (9) under the assumption that all the system states and time delay are available for the design, such that the corresponding closed loop system is asymptotically stable in the presence of time delay and uncertainties, with focus on disturbance tolerability but of convenient parameter design methodology.

\section{System analysis and basic assumptions}

In this section, a sliding surface will be designed for system (9) and the stability of corresponding sliding motion will be analysed. First, it is necessary to impose the following fundamental assumptions on the system (9).

Assumption 1: The matrix pair $(A, B)$ is controllable.

From Section 2, it follows that under Assumption 1, there exists new coordinates

$$
z=T x
$$

such that in the new coordinates $z$, the system (9) can be described by

$$
\begin{aligned}
\dot{z}_{1}= & A_{11} z_{1}+A_{d 11} z_{1}(t-d)+A_{12} z_{2}+A_{d 12} z_{2}(t-d) \\
& +F_{1}\left(t, z, z_{d}\right) \\
\dot{z}_{2}= & A_{21} z_{1}+A_{d 21} z_{1}(t-d)+A_{22} z_{2}+A_{d 22} z_{2}(t-d) \\
& +B_{2} u(t)+B_{2} G\left(t, z, z_{d}\right)+F_{2}\left(t, z, z_{d}\right)
\end{aligned}
$$

where $z(t)=\operatorname{col}\left(z_{1}, z_{2}\right)$ with $z_{1} \in \mathfrak{R}^{n-m}$ and $z_{2} \in \mathfrak{R}^{m}$, $z_{d}=\operatorname{col}\left(z_{1}(t-d) ; z_{2}(t-d)\right)$ with $z_{1}(t-d) \in \mathfrak{R}^{n-m}$ and $z_{2}(t-d) \in \mathfrak{R}^{m}$, and

$$
\begin{aligned}
T A T^{-1} & =\left[\begin{array}{ll}
A_{11} & A_{12} \\
A_{21} & A_{22}
\end{array}\right] \\
T A_{d} T^{-1} & =\left[\begin{array}{ll}
A_{d 11} & A_{d 12} \\
A_{d 21} & A_{d 22}
\end{array}\right], T B=\left[\begin{array}{c}
0 \\
B_{2}
\end{array}\right]
\end{aligned}
$$

where $\quad A_{11}, \quad A_{d 11} \in \mathfrak{R}^{(n-m) \times(n-m)}, \quad A_{12}, \quad A_{d 12} \in \mathfrak{R}^{(n-m) \times m}$, $A_{21}, \quad A_{d 21} \in \mathfrak{R}^{m \times(n-m)}, \quad A_{22}, \quad A_{d 22} \in \mathfrak{R}^{m \times m}, B_{2} \in \mathfrak{R}^{m \times m} \quad$ is non-singular, and

$$
\begin{aligned}
& G\left(t, z(t), z_{d}(t)\right)=\left.\bar{G}\left(t, x, x_{d}\right)\right|_{x=T^{-1} z} \\
& {\left[\begin{array}{l}
F_{1}\left(t, z(t), z_{d}\right) \\
F_{2}\left(t, z(t), z_{d}\right)
\end{array}\right]:=\left.T \bar{F}\left(t, x(t), x_{d}\right)\right|_{x=T^{-1} z}}
\end{aligned}
$$

where $F_{1}(\cdot) \in \mathfrak{R}^{n-m}$ and $F_{2}(\cdot) \in \mathfrak{R}^{m}$. Specifically, the matrix pair $\left(A_{11}, A_{12}\right)$ is controllable and thus there exists matrix $M \in \mathfrak{R}^{m \times(n-m)}$ such that

$$
A_{11}-A_{12} M
$$

is asymptotically stable. Therefore, there exists $P>0$ such that

$$
\left(A_{11}-A_{12} M\right)^{T} P+\left(A_{11}-A_{12} M\right) P<0
$$

Assumption 2: The uncertain terms $G(\cdot), F_{1}(\cdot)$ and $F_{2}(\cdot)$ in (13) satisfy:

$$
\begin{aligned}
& \left\|G\left(t, z(t), z_{d}\right)\right\| \leq \phi\left(t, z(t), z_{d}\right) \\
& \left\|F_{1}\left(t, z(t), z_{d}\right)\right\| \leq \rho_{1}\left(t, z(t), z_{d}\right) \\
& \left\|F_{2}\left(t, z(t), z_{d}\right)\right\| \leq \rho_{2}\left(t, z(t), z_{d}\right)
\end{aligned}
$$

where $\phi(\cdot), \rho_{1}(\cdot)$ and $\rho_{2}(\cdot)$ are known nonnegative continuous functions.

Remark 1. Assumption 2 holds if the uncertainties $\bar{G}(\cdot)$ and $\bar{F}(\cdot)$ in (9) are bounded by known nonnegative continuous functions. In this paper, the bounds on $G(\cdot)$ and $F_{2}(\cdot)$ are assumed to be known which will be used in control design in order to enhance the robustness against the corresponding uncertainties. 


\section{Sliding motion analysis and control design}

Based on the assumptions in Section 4, the main aim of this section is to achieve robust stability of system (12)-(13) in the presence of disturbances and delay in using sliding mode control which guarantees a sliding motion occurs in finite time. From Section 2, it follows that under Assumption 1, the sliding function is defined as

$$
\sigma(z)=M z_{1}(t)+z_{2}(t)
$$

where $M \in \mathfrak{R}^{m \times(n-m)}$ is a designed matrix which satisfies (16). When the system is limited to the sliding surface

$$
\sigma(z)=0
$$

it follows that $z_{2}=-M z_{1}$.

From the structure of system (12)-(13), the sliding motion of system (9) associated with the sliding surface (21) is dominated by system (12). When dynamic (12) is limited to the sliding surface (21), it can be described by

$$
\begin{aligned}
\dot{z}_{1} & =\left(A_{11}-A_{12} M\right) z_{1}+\left(A_{d 11}-A_{d 12} M\right) z_{1}(t-d) \\
& +F_{1 \delta}\left(t, z_{1}(t), z_{1 d}\right)
\end{aligned}
$$

where

$$
F_{1 \delta}\left(t, z_{1}, z_{1 d}\right)=\left.F_{1}\left(t, z, z_{d}\right)\right|_{z_{2}=-M z_{1}}
$$

with $z=\operatorname{col}\left(z_{1}, z_{2}\right)$ and $F_{1}(\cdot)$ defined in (12).

Remark 2. System (22) is the sliding mode of system (12)-(13) corresponding to the sliding surface (21). It should be noted that the mismatched uncertainty $F_{1 \delta}(\cdot)$ is the uncertainty $F_{1}(\cdot)$ when it is limited to the sliding surface (21).

From equation (22) it is clear to see that the mismatched uncertainty $F_{1 \delta}(\cdot)$ can affect the sliding mode dynamics and as such it is necessary to impose some constraint on it in order to guarantee asymptotic stability of the sliding motion.

Assumption 3: There exist known function $\mu(\cdot)$ such that the uncertainty $F_{1 \delta}(\cdot)$ in (22) satisfies

$$
F_{1 \delta}\left(t, z, z_{d}\right) \leq \mu(t, z)\left\|z_{d}\right\|
$$

The following results are ready to be presented.

\subsection{Stability analysis of sliding motion}

Theorem 1: Under Assumptions 1 and 3, the sliding motion of system (12)-(13) associated with the sliding surface (21), governed by system (22) is uniformly asymptotically stable if there exist a scalar $\epsilon>0$ and a real positive definite matrix $P$ such that the inequality

$$
\begin{aligned}
& \lambda_{\min }\left(A_{o}^{T} P+P A_{o}\right)-k-\lambda_{\max }\left(P A_{1} P^{-1} A_{1}^{T} P\right) \\
& -2 \sqrt{k} \mu(\cdot) \lambda_{\max }(P)>0
\end{aligned}
$$

holds, where $k=\frac{(1+\epsilon) \lambda_{\max }(P)}{\lambda_{\min }(P)}, \epsilon>0$ and $\mu(\cdot)$ is a known non-negative function.
Proof: For sliding mode (22), consider the candidate Lyapunov function

$$
V_{1}=z_{1}^{T}(t) P z_{1}(t)
$$

Then the time derivative of $V_{1}$ along the trajectory of the system (22), is given by

$$
\begin{aligned}
\left.\dot{V}_{1}\right|_{(22)} & =z_{1}^{T}(t)\left(A_{o}^{T} P+P A_{o}\right) z_{1}(t)+2 z_{1}^{T} P A_{1} z_{1}(t-d) \\
& +2 z_{1}^{T}(t) P F_{1 \delta}\left(t, z_{1}(t), z_{1}(t-d)\right)
\end{aligned}
$$

where $A_{1}=A_{d 11}-A_{d 12} M$ and $F_{1 \delta}$ is defined in (23).

From Lemma 2, it follows that

Comment [a1]: Author: Please confirm if this is correct.

$$
\begin{aligned}
& 2 z_{1}^{T}(t) P A_{1} z_{1}(t-d) \leq z_{1}^{T}(t-d) P z_{1}(t-d) \\
& +z_{1}^{T}(t) P A_{1} P^{-1} A_{1}^{T} P z_{1}(t)
\end{aligned}
$$

From (27) and (28) it is observed that the derivative $V_{1}$ along the trajectory of system (22) can be described by

$$
\begin{aligned}
& \left.\dot{V}_{1}\right|_{(22)}=z_{1}^{T}(t)\left[A_{o}^{T} P+P A_{o}\right] z_{1}(t)+z_{1}^{T}(t-d) P z_{1}(t-d) \\
& +z_{1}^{T}(t) P A_{1} P^{-1} A_{1}^{T} P z_{1}(t)+2 z_{1}^{T} P F_{1 \delta}\left(t, z_{1}(t), z_{1}(t-d)\right)
\end{aligned}
$$

Applying the Razumikhin condition (see Lemma 1), for some positive constant $q=(1+\epsilon)$ with $\epsilon>0$, the following inequality holds:

$$
z_{1}^{T}(t-d) P z_{1}(t-d) \leq(1+\epsilon) z_{1}^{T}(t) P z_{1}(t)
$$

From (30), it follows that

$$
\begin{aligned}
& \lambda_{\min }(P)\left\|z_{1}(t-d)\right\|^{2} \leq z_{1}^{T}(t-d) P z_{1}(t-d) \\
& \leq(1+\epsilon) \lambda_{\max }(P)\left\|z_{1}\right\|^{2}
\end{aligned}
$$

Thus

$$
\left\|z_{1}(t-d)\right\|^{2} \leq k\left\|z_{1}\right\|^{2}
$$

where $k$ is defined in (25). From (24), (29) and (32),

$$
\begin{aligned}
& \left.\dot{V}_{1}\right|_{(22)} \leq-\lambda_{\min }(Q)\left\|z_{1}\right\|^{2}+k\left\|z_{1}\right\|^{2} \\
& +\lambda_{\max }\left(P A_{1} P^{-1} A_{1}^{T} P\right)\left\|z_{1}\right\|^{2}+2 \sqrt{k} \mu \lambda_{\max }(P)\left\|z_{1}\right\|^{2} \\
& =-\left(\lambda_{\min }(Q)-k-\lambda_{\max }\left(P A_{1} P^{-1} A_{1}^{T} P\right)\right. \\
& \left.-2 \sqrt{k} \mu(\cdot) \lambda_{\max }(P)\right)\left\|z_{1}\right\|^{2}
\end{aligned}
$$

From (25), it follows that $\dot{V}$ is negative definite. Hence the result follows.

Remark 3. Theorem 1 gives a sufficient condition which guarantee the asymptotic stability of the designed sliding motion. However, the left hand side of the inequality (25) is a function due to $\mu(\cdot)$, and thus it is difficult to obtain the design parameters to complete sliding mode design. In order to make the parameters more accessible, the LMI technique is used based on the following assumption.

Assumption 4: There exist known constants ${ }_{1}$ and $\quad 2$ such that the uncertainty $F_{1}(\cdot)$ in (12) satisfies

$$
F_{1}^{T}\left(t, z(t), z_{d}\right) F_{1}\left(t, z(t), z_{d}\right) \leq \varpi_{1}^{2} z^{T}(t)+\varpi_{2}^{2} z_{d}^{T} z_{d}
$$


Remark 4. Assumptions 2 and 3 are limitations to the nonlinear uncertainties. The bounds on $G(\cdot)$ and $F_{2}(\cdot)$ in (17) and (19) have general nonlinear form and involve time delay. In order to use LMI techniques to obtain the design parameters for sliding surface design, Assumption 3 is imposed on the mismatched uncertainty $F_{1}(\cdot)$ to facilitate the sliding motion analysis. However, it is not required that the bounds on uncertainty $\bar{F}$ in (9) satisfies linear growth condition. This is in comparison with many existing work (Gao et al., 2014; Qi et al., 2017; Hua et al., 2008; Xu, 1997) where sliding mode techniques are employed and (Ramakrishnan and Ray, 2015; Wang et al., 2016) where LMI is used.

From (34) and (23), it follows that

$$
\begin{aligned}
& F_{1 \delta}^{T}(\cdot) F_{1 \delta}(\cdot) \leq \varpi_{1}^{2}\left[z_{1}^{T}-\left(M z_{1}\right)^{T}\right]\left[\begin{array}{c}
z_{1} \\
-M z_{1}
\end{array}\right] \\
& +\varpi_{2}^{2}\left(\left[z_{1}^{T}(t-d)-\left(M z_{1}(t-d)\right)^{T}\right]\left[\begin{array}{c}
z_{1}(t-d) \\
-M z_{1}(t-d)
\end{array}\right]\right) \\
& =\varpi_{1}^{2}\left[z_{1}^{T} z_{1}+z_{1}^{T}\left(M^{T} M\right) z_{1}\right]+\varpi_{2}^{2}\left[z_{1}^{T}(t-d) z_{1}(t-d)\right. \\
& \left.+z_{1}(t-d)\left(M^{T} M\right) z_{1}(t-d)\right] \\
& \leq \varpi_{1}^{2}\left(1+\lambda_{\max }\left(M^{T} M\right)\right) z_{1}^{T} z_{1} \\
& +\varpi_{2}^{2}\left(1+\lambda_{\max }\left(M^{T} M\right)\right) z_{1}^{T}(t-d) z_{1}(t-d) \\
& \leq \psi_{1} z_{1}^{T} z_{1}+\psi_{2} z_{1}^{T}(t-d) z_{1}(t-d)
\end{aligned}
$$

where

$$
\begin{aligned}
& \psi_{1}=\varpi_{1}^{2}\left[1+\lambda_{\max }\left(M^{T} M\right)\right] \\
& \psi_{2}=\varpi_{2}^{2}\left[1+\lambda_{\max }\left(M^{T} M\right)\right]
\end{aligned}
$$

where $\quad 1$ and $\quad 2$ are constants satisfying (34).

Theorem 2: Under Assumptions 1 and 3, the sliding motion of system (12)-(13) associated with the sliding surface (21), governed by system (22) is uniformly asymptotically stable if there exist scalars $\alpha>0, \epsilon>0$ and a real positive definite matrix $P$ such that the following LMI holds

$$
\left[\begin{array}{cc}
W_{o} & P \\
P & -\alpha I
\end{array}\right]<0
$$

where

$$
\begin{aligned}
& W_{o}=A_{o}^{T} P+P A_{o}+(1+\epsilon) P+P A_{1} P^{-1} A_{1}^{T} P+\beta \alpha I \\
& A_{o}=A_{11}-A_{12} M \\
& A_{1}=A_{d 11}-A_{d 12} M \\
& \beta=\psi_{1}+\psi_{2} k
\end{aligned}
$$

where $k, \psi_{1}$ and $\psi_{2}$ are defined in (25), (36) and (37) respectively, and $M$ is defined in (20).

Proof: Using Lyapunov function (26), the time derivative of $V_{1}$ along the trajectory of the system (22), is given by (27). Then from (35) and (32), it follows that

$$
\begin{aligned}
& F_{1 \delta}^{T}(\cdot) F_{1 \delta}(\cdot) \\
& \leq \psi_{1} z_{1}^{T}(t) z_{1}(t)+\psi_{2}\left\|z_{1}(t-d)\right\|^{2} \\
& \leq \psi_{1} z_{1}^{T}(t) z_{1}(t)+\psi_{2} k z_{1}^{T}(t) z_{1}(t) \\
& =\left(\psi_{1}+\psi_{2} k\right) z_{1}^{T}(t) z_{1}(t)
\end{aligned}
$$

where $\psi_{1}$ and $\psi_{2}$ are defined in (37).

Using (39), by similar analysis as in Theorem 1, the proof of the time derivative of $V_{1}(\cdot)$ is given by

$$
\begin{aligned}
\left.\dot{V}_{1}\right|_{(22)}= & \\
& z_{1}^{T}(t)\left[A_{o}^{T} P+P A_{o}+(1+\epsilon) P+P A_{1} P^{-1} A_{1}^{T} P\right] z_{1}(t) \\
& +2 z_{1}^{T} P F_{1 \delta}\left[t, z_{1}(t), z_{1}(t-d)\right] \\
& =\left[\begin{array}{c}
z_{1}(t) \\
F_{1 \delta}
\end{array}\right]^{T}\left[\begin{array}{cc}
W & P \\
P & 0
\end{array}\right]\left[\begin{array}{c}
z_{1} \\
F_{1 \delta}
\end{array}\right]
\end{aligned}
$$

where

$$
W=A_{o}^{T} P+P A_{o}+(1+\epsilon) P+P A_{1} P^{-1} A_{1}^{T} P, \epsilon>0
$$

The inequality (39) can be rewritten as

$$
\left[\begin{array}{c}
z_{1}(t) \\
F_{1 \delta}
\end{array}\right]^{T}\left[\begin{array}{cc}
\beta I & 0 \\
0 & -I
\end{array}\right]\left[\begin{array}{c}
z_{1} \\
F_{1 \delta}
\end{array}\right] \geq 0
$$

where $\beta=\psi_{1}+\psi_{2} k$.

It can be seen from (40) and (42) that,

$$
\begin{aligned}
\left.\dot{V}_{1}\right|_{(22)} & \leq\left[\begin{array}{c}
z_{1}(t) \\
F_{1 \delta}
\end{array}\right]^{T}\left(\left[\begin{array}{cc}
W & P \\
P & 0
\end{array}\right]+\alpha\left[\begin{array}{cc}
\beta I & 0 \\
0 & -I
\end{array}\right]\right)\left[\begin{array}{c}
z_{1}(t) \\
F_{1 \delta}
\end{array}\right] \\
& =\left[\begin{array}{c}
z_{1}(t) \\
F_{1 \delta}
\end{array}\right]^{T}\left[\begin{array}{cc}
W+\beta \alpha I & P \\
P & -\alpha I
\end{array}\right]\left[\begin{array}{c}
z_{1} \\
F_{1 \delta}
\end{array}\right] \\
& =\left[\begin{array}{c}
z_{1}(t) \\
F_{1 \delta}
\end{array}\right]^{T}\left[\begin{array}{cc}
W_{o} & P \\
P & -\alpha I
\end{array}\right]\left[\begin{array}{c}
z_{1} \\
F_{1 \delta}
\end{array}\right]
\end{aligned}
$$

where $\alpha$ is a positive constant, $W_{o}$ and $W$ are defined by (38) and (41) respectively. By applying inequality (38) to (43), it follows that $\dot{V}$ is negative definite. Hence the result follows.

Remark 5. It should be noted that the inequality in (25) in Theorem 1 involves function $\mu(\cdot)$ which makes it difficult to determine the design parameters, although Theorem 1 is less conservative. In connection with this, a set of conditions has been expressed in LMI to guarantee the stability of sliding motion and thus, the associated design parameters can be obtained systematically using LMI techniques. This is in comparison with the work (Yan et al., 2010).

Remark 6. From the proof of Theorem 2, it follows that it is unnecessary to assume that the bound on the uncertainty $F_{1}\left(t, z(t), z_{d}(t)\right)$ has the special form in (34). Actually, it is only required that the bound on $F_{1 \delta}(\cdot)$ defined in (35) has the special form in (34). Therefore, in this paper, the requirement on the bound on mismatched uncertainty is relaxed which is allowed to have more general form. 


\subsection{Sliding mode control design}

The objective now is to design a state feedback sliding mode control law such that the system state is driven to the sliding surface (21) in finite time. The following control is proposed:

$$
\begin{aligned}
u(t) & =-B_{2}^{-1}\left(\Gamma+\left\{\left\|B_{2}\right\| \phi\left(t, z(t), z_{d}\right)+\|M\| \rho_{1}(\cdot)\right.\right. \\
& \left.\left.+\rho_{2}(\cdot)+\eta\right\} \operatorname{sgn}(\sigma(z))\right)
\end{aligned}
$$

where

$$
\begin{aligned}
\Gamma= & \left(M A_{11}+A_{21}\right) z_{1}(t)+\left(M A_{d 11}+A_{d 21}\right) z_{1}(t-d) \\
& +\left(M A_{12}+A_{22}\right) z_{2}(t)+\left(M A_{d 21}+A_{d 22}\right) z_{2}(t-d)
\end{aligned}
$$

$\phi(\cdot), \rho_{1}(\cdot)$ and $\rho_{2}(\cdot)$ in (17)-(19) are defined respectively and $\eta>0$ is the reachability constant. The following result is ready to be presented.

Theorem 3: Consider the system (12)-(13). The control (44) drives the system (12)-(13) to the sliding surface (21) in finite time and maintains a sliding motion on it thereafter.

Proof: From (20) and (12)-(13), it can be verified that

$$
\begin{aligned}
\dot{\sigma}(z) & =M\left(A_{11} z_{1}+A_{d 11} z_{1}(t-d)\right. \\
& \left.+A_{12} z_{2}+A_{d 12} z_{2}(t-d)+F_{1}\left(t, z(t), z_{d}\right)\right) \\
& +\left(A_{21} z_{1}+A_{d 21} z_{1}(t-d)+A_{22} z_{2}\right. \\
& \left.+A_{d 22} z_{2}(t-d)\right)+B u(t)+B G\left(t, z(t), z_{d}\right) \\
& \left.+F_{2}\left(t, z(t), z_{d}\right)\right) \\
& =\Gamma+B u(t)+B G\left(t, z(t), z_{d}\right)+M F_{1}\left(t, z(t), z_{d}\right) \\
& +F_{2}\left(t, z(t), z_{d}\right)
\end{aligned}
$$

where $\Gamma$ is defined in (45).

Applying the control $u$ in (44) to (46), it follows from (17) and (19),

$$
\begin{aligned}
& \sigma^{\tau} \dot{\sigma}=\sigma^{\tau}(z) \Gamma-\sigma^{\tau}(z) M F_{1}\left(t, z(t), z_{d}\right) \\
& -\sigma^{\tau}(z)\left[\Gamma+\left\{\|M\| \rho_{1}\left(t, z(t), z_{d}\right)+\|B\| \phi\left(t, z(t), z_{d}\right)\right.\right. \\
& \left.+\rho_{2}\left(t, z(t), z_{d}\right)+\eta\right\} \operatorname{sgn}(\sigma)+B G\left(t, z(t), z_{d}\right) \\
& +F_{2}\left(t, z(t), z_{d}\right) \\
& =\sigma^{\tau}(z) M F_{1}\left(t, z(t), z_{d}\right)-\|\sigma(z)\|\|M\| \rho_{1}\left(t, z(t), z_{d}\right) \\
& +\sigma^{\tau}(z) B G\left(t, z(t), z_{d}\right)-\|\sigma\|\|B\| \phi\left(t, z(t), z_{d}\right) \\
& +\sigma^{\tau}(z) F_{2}\left(t, z(t), z_{d}\right)-\|\sigma\| \rho\left(t, z(t), z_{d}\right)-\eta\|\sigma(z)\| \\
& \leq\left\|\sigma^{\tau}(z) M F_{1}\left(t, z(t), z_{d}\right)\right\|-\|\sigma(z)\|\|M\| \rho_{1}\left(t, z(t), z_{d}\right) \\
& +\left\|\sigma^{\tau}(z) B G\left(t, z(t), z_{d}\right)\right\|-\|\sigma\|\|B\| \phi\left(t, z(t), z_{d}\right) \\
& +\left\|\sigma^{\tau}(z) F_{2}\left(t, z(t), z_{d}\right)\right\|-\|\sigma\| \rho\left(t, z(t), z_{d}\right) \\
& \leq-\eta\|\sigma(z)\|
\end{aligned}
$$

where the fact that $\sigma^{\tau}(z) \operatorname{sgn}(\sigma(z)) \geq\|\sigma(z)\|$ (see Yan et al., 2010 ) is used to obtain the inequality above.

This shows that the reachability condition holds and hence the conclusion follows.
Theorems 1 and 2 together show that the corresponding closed-loop system is uniformly asymptotically stable.

Remark 7. It should be noted that the designed controller (44) is expressed in $z$ coordinates. The corresponding controller in $x$ coordinates is easy to obtain using the transformation $z=T x$ in (11) which can be obtained using basic matrix theory (Edwards and Spurgeo, 1998). The reachability analysis above is carried out directly in $z$ coordinates, which may reduce the conservatism. It should be noted that there is no limitation to the change rate of time varying delay $d(t)$. This is in comparison with many existing work (Jia et al., 2017).

\section{Application and simulation results}

Consider the cascaded CSTR system in Hua et al. (2009) which is used to illustrate the effectiveness of the developed method in this paper. The compositions $C_{A}$ and $C_{B}$ of the produce streams from reactor $\mathrm{A}$ and reactor $\mathrm{B}$, represents the system states which are to be controlled. The output of one reactor CSTR determines the flow rate into the second reactor and vice versa.

A time delay is added between the output of one reactor and the input (flow rate) of the other reactor such that at a certain time, the state of one reactor is determined by the state of the other reactor at a previous time $t-d(t)$. Refer to Hua et al. (2009) for more information on CSTR. By choosing the same parameters as in Hua et al. (2009), the mathematical model to describe the CSTR is given by

$$
\begin{aligned}
\dot{z}_{1}= & -z_{1}-0.5 z_{1}(t-d)+z_{2}+F_{1}\left(t, z, z_{d}\right) \\
\dot{z}_{2}= & z_{1}+z_{1}(t-d)-2.8333 z_{2}+z_{2}(t-d) \\
& +\left(u+G\left(t, z(t), z_{d}\right)\right)+F_{2}\left(t, z, z_{d}\right)
\end{aligned}
$$

where $z_{1}:=C_{A}-C_{A}^{*}, z_{2}:=C_{B}-C_{B}^{*}$ and $C_{A}^{*}=14 / 9$ and $C_{B}^{*}=7 / 3$ (see Hua et al., 2009).

It should be noted that the uncertainties $G(\cdot)$ and $F_{1}(\cdot)$ and $F_{2}(\cdot)$ are added in system (48)-(49) specifically to illustrate the obtained theoretical results, which are assumed to satisfy

$$
\begin{aligned}
& \left\|G\left(t, z(t), z_{d}\right)\right\| \leq \underbrace{5|\sin (t)|\left|z_{1}(t) z_{2}(t-d)\right|}_{\phi\left(t, z(t), z_{d}\right)} \\
& \left\|F_{2}\left(t, z(t), z_{d}\right)\right\| \leq \underbrace{5|\cos (t)|\left|z_{2}(t-d)\right|}_{\rho\left(t, z(t), z_{d}\right)}
\end{aligned}
$$

and $F_{1}(\cdot)$ satisfies

$$
F_{1}^{T} F_{1} \leq \underbrace{0.86^{2}}_{\sigma_{1}} z^{T} z_{d}+\underbrace{0.65^{2}}_{\sigma_{2}} z_{d}^{T} z_{d}
$$

Choose the sliding function $\sigma(z)=\left[\begin{array}{ll}2 & 1 \\ M & 1\end{array}\right] z$. By direct calculation, $A_{o}=-3, A_{1}=-0.5$. With $Q=I$ and $P=0.1667$ obtained by solving the LMI in (43), $=0: 07$, and $\alpha=5.5781$ is the maximum boundary which ensures that 


$$
\left[\begin{array}{cc}
W_{o} & P \\
P & -\alpha I
\end{array}\right]=\left[\begin{array}{cc}
-0.3995 & 0.1667 \\
0.1667 & -0.0700
\end{array}\right]<0
$$

Thus the matrix (38) is symmetric negative definite.

From the result above, it can be verified that all the conditions in Theorem 1 are satisfied. Thus from Theorem 1, the sliding motion associated with the sliding surface is asymptotically stable.

Figure 1 Time response of state variables $z_{1}(t)$ and $z_{2}(t)$ of system (48)-(49) (see online version for colours)

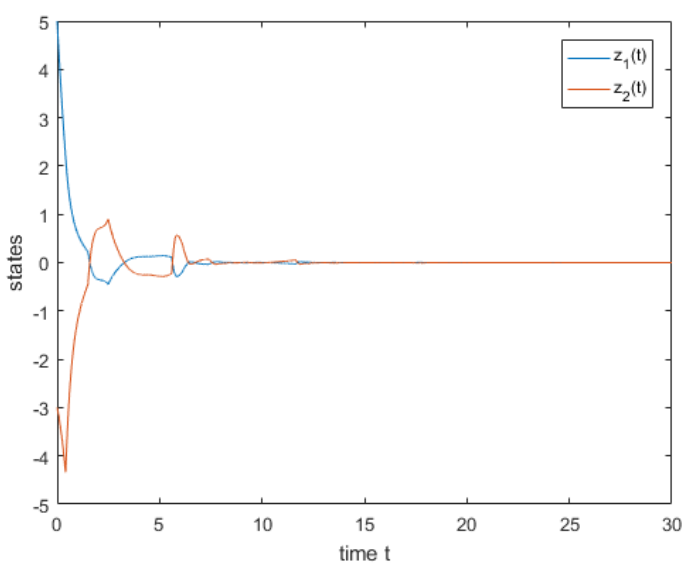

Figure 2 Time response of the control signal $u(t)$ and sliding function $\sigma(z)$ (see online version for colours)

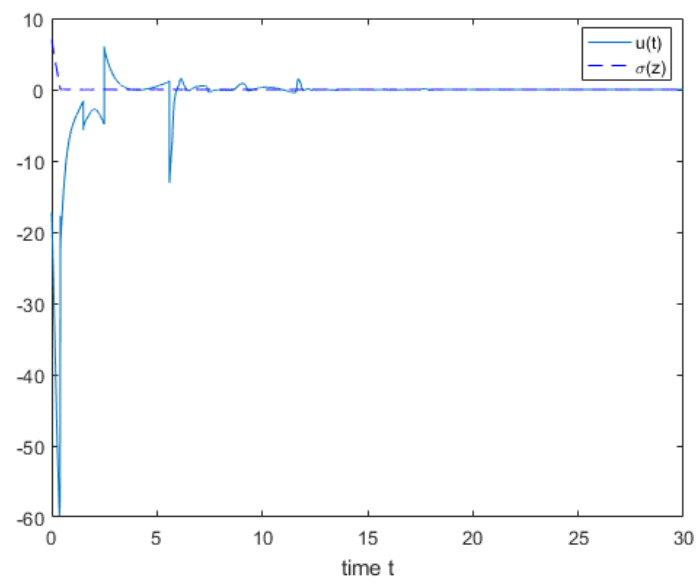

From Theorem 2 and by direct calculation, the sliding mode control law (44) given by

$$
\begin{aligned}
u= & -\Gamma\left(1.3778\|z(t)\|^{2}+0.845\left\|z_{d(t)}\right\|^{2}+1.5 \cos (t)\left|z_{d 2}\right|\right. \\
& \left.+5 \sin (t)\left|z_{1}(t) z_{d 2}\right|+2\right) \operatorname{sgn}(\sigma(z))
\end{aligned}
$$

stabilises the system (48)-(49), where

$$
\Gamma=-2 z_{1}(t)-0.833 z_{2}(t)+3 z_{2}(t-d)
$$

For simulation purposes, the initial condition relating to the time delay is chosen as $z(t)=\operatorname{col}\left(\sin (t)\right.$, $\left.\mathrm{e}^{t}\right)$ and the time delay is $d(t)=5-3 \sin t$. The time responses of the state variables and control signal are shown in Figures 1 and 2, respectively, which demonstrate that the proposed approach is effective.

\section{Conclusions}

In this paper, state feedback sliding mode control for a class of time delay systems has been considered, where time delay exists in both system states and disturbances. Conservatism is reduced by fully using the property that sliding mode dynamics are of reduced order, and using the Razumikhin approach, the developed results can accommodate the large time delays. Also, it has been shown that though the uncertainty bounds are nonlinear, they can be obtained using LMI technique. Sliding mode control has been designed to guarantee the systems reachability to the sliding surface, and the nonlinear bounds on uncertainties have been fully employed in control design. The results of the simulation verify the theoretical analysis and further illustrate the feasibility of the proposed methodology, through application to the control problem of the CSTR system. However, as systems become larger and more complicated, it is interesting to consider large time delays in interconnected systems in the future.

\section{Acknowledgements}

This paper is partially supported by the National Natural Science Foundation of China via grant 61573180 .

\section{References}

Baek, J., Jin, M. and Han, S. (2016) 'A new adaptive sliding-mode control scheme for application to robot manipulators', IEEE Transactions on Industrial Electronics, Vol. 63, No. 6, pp.3628-3637.

Cao, W-J. and Xu, J-X. (2004) 'Nonlinear integral-type sliding surface for both matched and unmatched uncertain systems', IEEE Transactions on Automatic Control, Vol. 49, No. 8, pp. $1355-1360$.

Chen, J., Zhu, Q.M., Li, J. and Liu, Y.J. (2017) 'Biased compensation recursive least squares-based threshold algorithm for time-delay rational models via redundant rule', Nonlinear Dynamics, Vol. 91, No. 2, pp.797-807.

Edwards, C. and Spurgeon, S. (1998) Sliding Mode Control: Theory and Applications, CRC Press, London.

Gao, Q., Feng, G., Liu, L., Qiu, J. and Wang, Y. (2014) 'An ISMC approach to robust stabilization of uncertain stochastic time-delay systems', IEEE Transactions on Industrial Electronics, Vol. 61, No. 12, pp.6986-6994.

Ghabi, J. (2018) 'A novel sliding mode controller scheme for a class of nonlinear uncertain systems', International Journal of Modelling, Identification and Control, Vol. 29, No. 2, pp.127-135. 
Gu, K., Chen, J. and Kharitonov, V.L. (2003) Stability of Time-Delay Systems, Springer Science \& Business Media, Boston.

Hua, C., Liu, P.X. and Guan, X. (2009) 'Backstepping control for nonlinear systems with time delays and applications to chemical reactor systems', IEEE Transactions on Industrial Electronics, Vol. 56, No. 9, pp.3723-3732.

Hua, C-C., Wang, Q-G. and Guan, X-P. (2008) 'Memoryless state feedback controller design for time delay systems with matched uncertain nonlinearities', IEEE Transactions on Automatic Control, Vol. 53, No. 3, pp.801-807.

Jia, X., Chen, X., Xu, S., Zhang, B. and Zhang, Z. (2017) 'Adaptive output feedback control of nonlinear time-delay systems with application to chemical reactor systems', IEEE Transactions on Industrial Electronics, Vol. 64, No. 6, pp.4792-4799.

Li, H., Wang, J., Wu, L., Lam, H-K. and Gao, Y. (2017) 'Optimal guaranteed cost sliding mode control of interval type-2 fuzzy time-delay systems', IEEE Transactions on Fuzzy Systems, Vol. 26, No. 1, pp.246-257.

Mu, J., Yan, X-G., Spurgeon, S.K. and Mao, Z. (2017) 'Nonlinear sliding mode control of a two-wheeled mobile robot system', International Journal of Modelling, Identification and Control, Vol. 27, No. 2, pp.75-83.

Nikkhah, M., Ashrafiuon, H.and Muske, K.R. (2006) 'Optimal sliding mode control for underactuated systems', in American Control Conference, IEEE, 6pp.

Qi, W., Park, J.H., Jun, C. and Kao, Y. (2017) 'Robust stabilization for nonlinear time-delay semi-Markovian jump systems via sliding mode control', IET Control Theory \& Applications, Vol. 11, No. 10, pp.1504-1513.

Ramakrishnan, K. and Ray, G. (2015) 'Stability criteria for nonlinearly perturbed load frequency systems with timedelay', IEEE Journal on Emerging and Selected Topics in Circuits and Systems, Vol. 5, No. 3, pp.383-392.

Richard, J-P. (2003) 'Time-delay systems: an overview of some recent advances and open problems', Automatica, Vol. 39 No. 10, pp.1667-1694.

Vaidyanathan, S. and Rhif, A. (2017) 'A novel four-leaf chaotic system, its control and synchronisation via integral sliding mode control', International Journal of Modelling, Identification and Control, Vol. 28, No. 1, pp.28-39.
Wang, X., Zong, G. and Sun, H. (2016) 'Asynchronous finite-time dynamic output feedback control for switched time-delay systems with non-linear disturbances', IET Control Theory \& Applications, Vol. 10, No. 10, pp.1142-1150.

Wu, X., Li, Y., Zhang, J. and Zhu, Q. (2014) 'Sliding mode control for neutral systems with uncertain parameters', International Journal of Modelling, Identification and Control, Vol. 21, No. 1, pp.65-71.

Xu, B. (1997) 'Stability robustness bounds for linear systems with multiple time-varying delayed perturbations', International Journal of Systems Science, Vol. 28, No. 12, pp.1311-1317.

Yan, X-G., Spurgeon, S.K. and Edwards, C. (2010) 'Static output feedback sliding mode control for time-varying delay systems with time-delayed nonlinear disturbances', International Journal of Robust and Nonlinear Control, Vol. 20, No. 7, pp.777-788.

Yan, X-G., Spurgeon, S.K. and Edwards, C. (2012) 'Global decentralised static output feedback slidingmode control for interconnected time-delay systems', IET Control Theory \& Applications, Vol. 6, No. 2, pp.192-202.

Yan, X-G., Spurgeon, S.K. and Edwards, C. (2014a) 'Memoryless static output feedback sliding mode control for nonlinear systems with delayed disturbances', IEEE Transactions on Automatic Control, Vol. 59, No. 7, pp.1906-1912.

Yan, X-G., Zhang, Q., Spurgeon, S.K., Zhu, Q. and Fridman, L.M. (2014b) 'Decentralised control for complex systems - an invited survey', International Journal of Modelling, Identification and Control, Vol. 22, No. 4, pp.285-297.

Yan, X-G., Spurgeon, S.K. and Edwards, C. (2017) Variable Structure Control of Complex Systems: Analysis and Design, Springer, Cham, Switzerland.

Zhao, D., Zhu, Q. and Dubbeldam, J. (2015) 'Terminal sliding mode control for continuous stirred tank reactor', Chemical Engineering Research and Design, Vol. 94, pp.266-274.

Zhen, R., Chen, J., Wu, X., Zhu, Q. and Nouri, H. (2014) 'Sliding mode control of uncertain switch systems with time-delay and disturbance', International Journal of Modelling, Identification and Control, Vol. 21, No. 4, pp.362-369. 